\title{
Characterization and evolution of dermal filaments from patients with Morgellons disease
}

This article was published in the following Dove Press journal:

Clinical, Cosmetic and Investigational Dermatology

7 January 2013

Number of times this article has been viewed

\section{Marianne J Middelveen' \\ Peter J Mayne' \\ Douglas G Kahn² \\ Raphael B Stricker'}

'International Lyme and Associated Diseases Society, Bethesda, MD, USA; ${ }^{2}$ Department of Pathology, Olive View-UCLA Medical Center, Sylmar, CA, USA

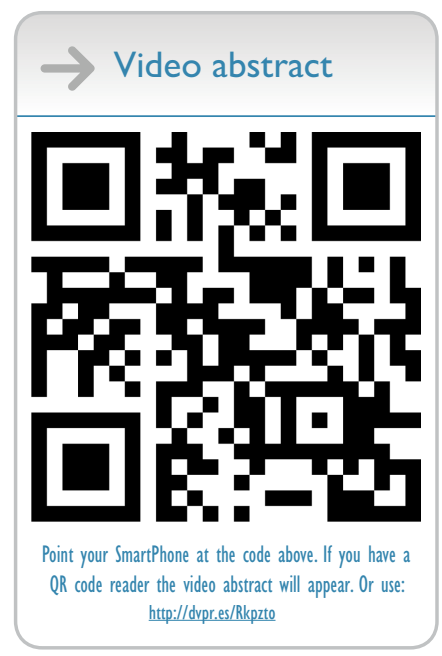

Correspondence: Raphael B Stricker 450 Sutter Street, Suite 1504, San Francisco, CA 94108, USA

Tel + I 4I5 3991035

Fax + I 4153991057

Email rstricker@usmamed.com
Abstract: Morgellons disease is an emerging skin disease characterized by formation of dermal filaments associated with multisystemic symptoms and tick-borne illness. Some clinicians hypothesize that these often colorful dermal filaments are textile fibers, either self-implanted by patients or accidentally adhering to lesions, and conclude that patients with this disease have delusions of infestation. We present histological observations and electron microscopic imaging from representative Morgellons disease samples revealing that dermal filaments in these cases are keratin and collagen in composition and result from proliferation and activation of keratinocytes and fibroblasts in the epidermis. Spirochetes were detected in the dermatological specimens from our study patients, providing evidence that Morgellons disease is associated with an infectious process.

Keywords: Morgellons disease, digital dermatitis, Lyme disease, Borrelia burgdorferi, spirochetes, keratin, keratinocytes, collagen, fibroblasts

\section{Introduction}

Morgellons disease (MD) is an emerging skin disease characterized by production of unusual filaments beneath unbroken skin or projecting from spontaneously appearing, slowly healing skin lesions in conjunction with often debilitating multisystemic symptoms. ${ }^{1,2} \mathrm{MD}$ is clinically associated with positive serology to Borrelia burgdorferi and/or a Lyme disease diagnosis. ${ }^{3}$ There are many similarities between MD and bovine digital dermatitis (BDD), an emerging disease of dairy cattle. ${ }^{4,5}$ BDD skin lesions are frequently found above the heel bulb of hind feet, ${ }^{6}$ and chronic proliferative-stage BDD is characterized by abnormal filament formation. In this stage, lesions demonstrate parakeratotic hyperkeratosis, acanthosis, ulcerated dermal papillae tips, and elongation of keratinocytes evolving into long keratin filaments. ${ }^{7-11}$ Various Treponema spp have been associated with BDD. ${ }^{7-11}$

Like the filaments seen in BDD skin lesions, MD filaments are reported to be composed of keratin and the products of keratinocytes. ${ }^{4,5}$ Filaments associated with MD lesions exhibit physical and chemical properties consistent with keratin, and the keratin content of filaments was confirmed by immunohistochemical staining with keratin-specific monoclonal antibodies. ${ }^{4,5}$ MD filaments were reported to originate from both pavement epithelial tissue and hair follicles that are predominantly populated by keratinocytes. ${ }^{4,5}$

Some clinicians have attributed MD to delusions of parastitosis or delusional infestation, claiming that filaments are self-implanted textile fibers. ${ }^{12-16}$ The fact that MD filaments frequently exhibit brilliant coloration that is unfamiliar in mammalian tissue may 
contribute to the misperception that the filaments are textile in nature. Coloration may include blue, red, green, and purple, although filaments may also be white or black. ${ }^{1,2,4}$ Filaments, regardless of coloration, have been shown to stem from follicular sheaths, and hyaline filaments have been shown to stem from the stratum basale. ${ }^{5}$ Some colored filaments have follicular bulbs and scaling consistent with the morphology of hairs, although others do not. This implies that at least some colored MD fibers and some hyaline fibers are abnormal hairs. ${ }^{5}$

The basis of unusual color presentation of MD filaments is not yet fully understood, but was hypothesized to be structural coloration rather than pigmentation. ${ }^{4,5}$ Bluish coloration of skin has been described in patients with pinta, a tropical disease caused by the spirochete Treponema carateum. ${ }^{17}$ This observation suggests that altered melanin expression could be responsible for the blue and red coloration of MD fibers. ${ }^{18}$ To address unanswered questions about MD filaments, we undertook a histological and electron microscopic study of representative MD tissue samples in order to gain insight into the evolution and composition of these filaments.

\section{Materials and methods} Human and bovine samples

Nonbiopsy dermatological specimens representative of MD lesions were collected from four MD subjects - two from the US (patients 1 and 2), and two from Canada (patients 3 and 4) - who met the key clinical criteria for MD. Patients 1 and 2 were previously presented as case studies. ${ }^{5}$ Patient 3 was not under the care of a physician at the time of this study, and patient 4 was referred to the authors of this study by a Canadian dermatologist. Fibers associated with these patients' lesions were embedded under unbroken skin or projected from dermatological tissue. Calluses with attached filaments were collected from all four patients after informed consent was obtained.

Biopsies from cattle with BDD were kindly provided by Dr Dorte Dopfer, Faculty of Veterinary Medicine, University of Wisconsin, Madison, WI, USA for comparison with MD dermatological specimens.

\section{Gross morphology}

The collected Morgellons samples were examined by brightfield microscopy at 40× and $100 \times$ magnification, illuminated either superior to the specimen to observe gross morphological characteristics or under a dissecting microscope at $8 \times$. BDD biopsies were examined with a dissecting microscope at $8 \times$ magnification to observe gross morphological characteristics. Only specimens that had filaments attached to epithelial tissue were included in this study, in order to avoid accidental contamination with extraneous textile fibers. Unattached filaments were excluded.

\section{Histology}

Human and bovine samples were formalin-fixed and embedded in paraffin for histological studies. Representative sections of formalin-fixed, paraffin-embedded MD samples were stained for the detection of keratin, collagen, melanin, and spirochetes. Immunohistochemical detection of keratin using monoclonal antibodies CK AE1/AE3 (pan-cytokeratin 1/3) and CK AE5/AE6 (restricted to cytokeratin 5/6) was performed by both Interscope Pathology Medical Group, Canoga Park, CA, USA and McClain Laboratory, Smithtown, NY, USA. Fontana-Masson staining (to detect melanin) and Gömöri trichrome staining (to detect collagen and keratin) were performed by McClain Laboratories. Warthin-Starry staining, performed by Interscope Pathology Medical Group, and Dieterle staining, performed by McClain Laboratory, were used to detect the presence of spirochetes.

BDD samples were sent for processing to Prairie Diagnostic Services, Saskatoon, SK, Canada, and samples were stained for spirochetes using Warthin-Faulkner stain and for keratin using CK AE1/AE3 stain. The BDD samples had additional staining for keratin using CK AE5/AE6 by Calgary Laboratory Services, Calgary, AB, Canada.

\section{Electron microscopy}

Morgellons and BDD samples were fixed in buffered 2.5\% glutaraldehyde. Scanning electron microscopy (SEM) and transmission electron microscopy (TEM) were performed by the Electron Microscopy Facility, Department of Materials Science and Engineering, Clemson University, Anderson, SC, USA, according to the protocols below.

\section{SEM}

Glutaraldehyde-fixed samples for SEM were washed in buffer and dehydrated in a graded series of ethanol concentrations. Samples were then immersed in hexamethyldisilazane for 5-15 minutes and air-dried at room temperature. Dried samples were mounted on Al mounts. Samples were not coated but placed into a Hitachi (Tokyo, Japan) TM3000 microscope and imaged in the variable pressure mode.

\section{TEM}

Glutaraldehyde-fixed samples were washed in buffer, followed by dehydration in a graded series of ethanol concentrations. Samples were then immersed in a 50:50 mixture of LR 
White embedding resin and $100 \%$ ethanol for 30 minutes, followed by pure LR White resin until samples settled on the bottom of the vial. The resin-immersed samples were then placed into pure resin in beam capsules and put into a $60^{\circ} \mathrm{C}$ oven overnight for polymerization. Sections were cut on an Ultracut E (Leica Microsystems, Wetzlar, Germany) microtome to produce sections $60-90 \mathrm{~nm}$ thick, placed onto copper grids and stained in uranyl acetate for 20 minutes. Images were taken on a Hitachi 7600 microscope.

\section{Results}

\section{Clinical observations}

The clinical features of our patients are shown in Table 1. All four patients were women, and their ages ranged from 49 to 73 years. Two patients resided in Texas, USA, and two patients were from Alberta, Canada. None of the patients had a history of psychosis or any evidence of delusional disease. All four patients had negative rapid plasma reagin testing for syphilis, and all four had positive serological testing for B. burgdorferi (Table 1). A summary of the histopathological and electron microscopic results described below is shown in Table 2.

\section{Microscopic observations Gross microscopic observations MD epithelial samples}

Calluses collected from patient 1 were carpeted in white or hyaline filaments attached to and projecting from one side of the callus (Figure 1A). Samples demonstrating floral or stellate structures with tentacle-like tips were also examined. Calluses removed from patients 2 and 3 demonstrated an external convex side (oriented to the external skin surface) and a concave side (oriented down towards the dermis). Colored (blue or red) or white filaments, $10-40 \mu \mathrm{m}$ in diameter, were more frequently found projecting out from the convex external surface, but some filaments were distributed within and throughout the callus (Figure 1B and C). Filaments on the concave side of the callus were rarely present.

Protruding keratin projections were observed on the concave, underside surface of calluses. Some of these were sharp at the tips of the projection, while others were blunt or ballooned (Figure 1D). Clear, ingrown hairs or hairlike structures, approximately $60 \mu \mathrm{m}$ in diameter, were observed protruding from the tips of some keratin projections. Patient 1 presented dermatological tissue with attached white filaments. In contrast, patient 4 had only small lesions without significant callus formation and presented a single chunk of dermatological tissue approximately $1 \mathrm{~mm}$ in diameter embedded with filaments rather than calluses.

\section{BDD biopsies}

BDD biopsies demonstrated chronic, late-stage, off-white, or grey filamentous projections on the external surface. In some samples, ingrowth of keratin on the interior surface was apparent.

\section{Histologic staining for keratin and collagen MD filaments}

Almost all the filaments from patients 1-4 that were cut longitudinally, obliquely, and in cross-section demonstrated a hollow medulla and surrounding cortex. The filaments were round, elliptical/elongated, bean-like, or curved. This morphology was consistently visible in histological sections (Figure 2A).

The presence of keratin was initially detected by CK AE1/ AE3 staining. The irregular, patchy CK AE1/AE3 staining of filamental sections suggested that the filaments might have a collagen content in addition to keratin (Figure 2B). Filaments were then stained with Gömöri trichrome to detect both keratin and collagen. The latter stains collagen green or bluish green and keratin red, thus allowing keratin and collagen to be differentiated.

Filaments stained in a similar manner regardless of original color, and no filaments were observed that did not take up stain. Filaments stained predominantly for collagen, and rarely stained exclusively for keratin. When positive keratin staining occurred, it was most often irregular or in patches (Figure 2C). Keratin staining was more pronounced when stained with CK AE1/AE3 or Gömöri trichrome.

Table I Summary of patient characteristics

\begin{tabular}{|c|c|c|c|c|c|c|}
\hline Subjects & Sex & Age, years & Area of residence & Delusional disease & RPR & Borrelia burgdorferi serology \\
\hline Patient I & Female & 72 & San Antonio, TX, USA & No & Negative & Positive \\
\hline Patient 2 & Female & 49 & Hughes Springs, TX, USA & No & Negative & Positive \\
\hline Patient 3 & Female & 54 & Cardston, AB, Canada & No & Negative & Positive \\
\hline Patient 4 & Female & 73 & Calgary, AB, Canada & No & Negative & Positive \\
\hline
\end{tabular}

Abbreviation: RPR, rapid plasma reagin. 


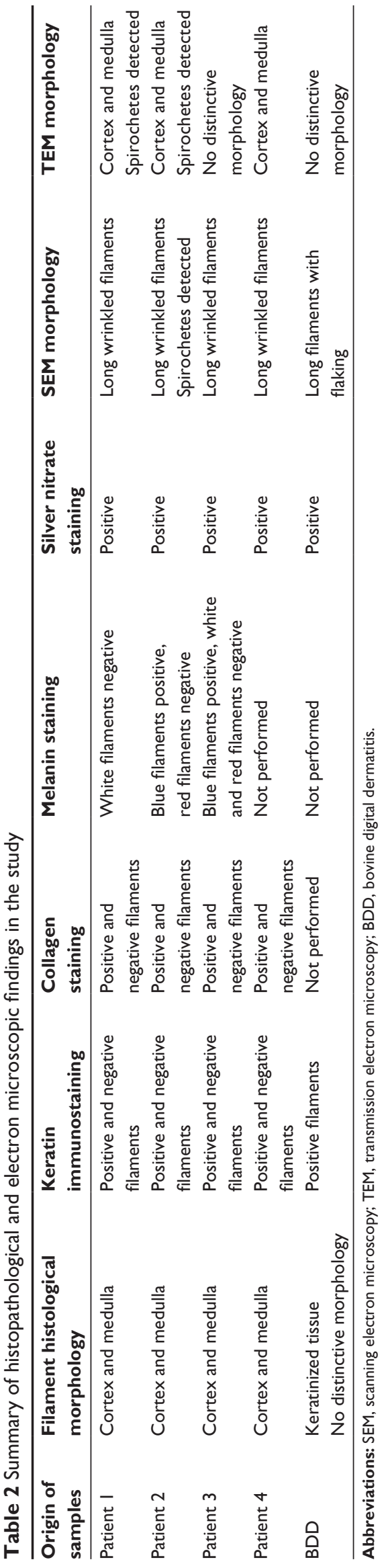

CK AE5/AE6 staining was negligible. Many filaments demonstrated retained nuclei (Figure 2D). Sectioned specimens of samples from patient 1 that revealed floral-like or stellate structures when examined for gross morphology demonstrated tentacle-like filaments likewise stemming from basal epithelial cells. These tentacle-like filaments also demonstrated retained nuclei (Figure 2E).

\section{MD calluses and other dermatological tissue}

Dermatological tissue from patients 1-4 demonstrated hyperplasia and parakeratotic hyperkeratosis. All dermatological specimens demonstrated strongly positive CK AE1/ AE3 staining for keratin and stained weakly with CK AE5/ AE6. Keratin staining was more pronounced in the stratum corneum. Calluses seen on sections from patient 1 demonstrated all the epidermal layers from the stratum corneum to the stratum basale. Filaments from this patient clearly stemmed from the stratum basale, with evolution inward towards the dermis (Figure 2F).

Filaments associated with the calluses collected from patients 2 and 3 commonly originated from collections of enlarged and/or elongated fibroblasts developing upwards toward the stratum corneum and projecting from the external surface (Figure 2G). Callus sections from patient 2 and to a lesser extent from patient 3 displayed pronounced conical to papillary shaped projections on the internal surface of the callus, and both CK AE1/AE3 and Gömöri trichrome staining demonstrated that these projections were keratin in composition. Masses of proliferative fibroblasts were contained within some of the keratin projections and stained positively for collagen (Figure $2 \mathrm{H}$ ). Some keratin projections appeared to have ruptured due to the pressure from fibroblast proliferation, and this occurred both towards the internal and external surfaces, but more frequently towards the external surface (Figure 2I). Filaments staining positively for collagen, both in longitudinal and cross-section, could be seen close to and within the collagen-positive staining fibroblast collections, suggesting fibroblast activation. Filament formation occurred mainly on the external side (Figure 2J).

\section{BDD sections}

BDD histological sections stained strongly with CK AE1/ AE3 (Figure 2K) and weakly with CK AE5/AE6 (Figure 2L) in a manner comparable to that of MD dermatological sections. The keratin staining of BDD filaments strongly resembled some of the hyperkeratotic, filamentous growth seen in MD histological sections. 


\section{Melanin detection by Fontana-Masson staining MD filaments}

Argentaffin and melanin granules are stained a blackish color with Fontana-Masson staining. Patient 1 had only hyaline filaments, and melanin staining of filament sections from this patient was negative. Patient 2 had some calluses with only blue filaments and some with only red filaments. Sections of blue filaments demonstrated positive melanin staining, while sections of red filaments lacked visible melanin staining. Patient 3 had calluses with red, white, and blue filaments; some of the filament sections from this patient were positive for melanin, while others were negative (Figure 3).

\section{Silver nitrate-based staining for spirochetes MD dermatological sections}

Warthin-Starry staining of MD dermatological sections from patients 1 and 2 revealed bacteria consistent with various morphological forms of Borrelia spp among epidermal cells (Figure 4A-C). These spiral or curved structures ranged from $0.1 \mu \mathrm{m}$ to $0.5 \mu \mathrm{m}$ in diameter and up to
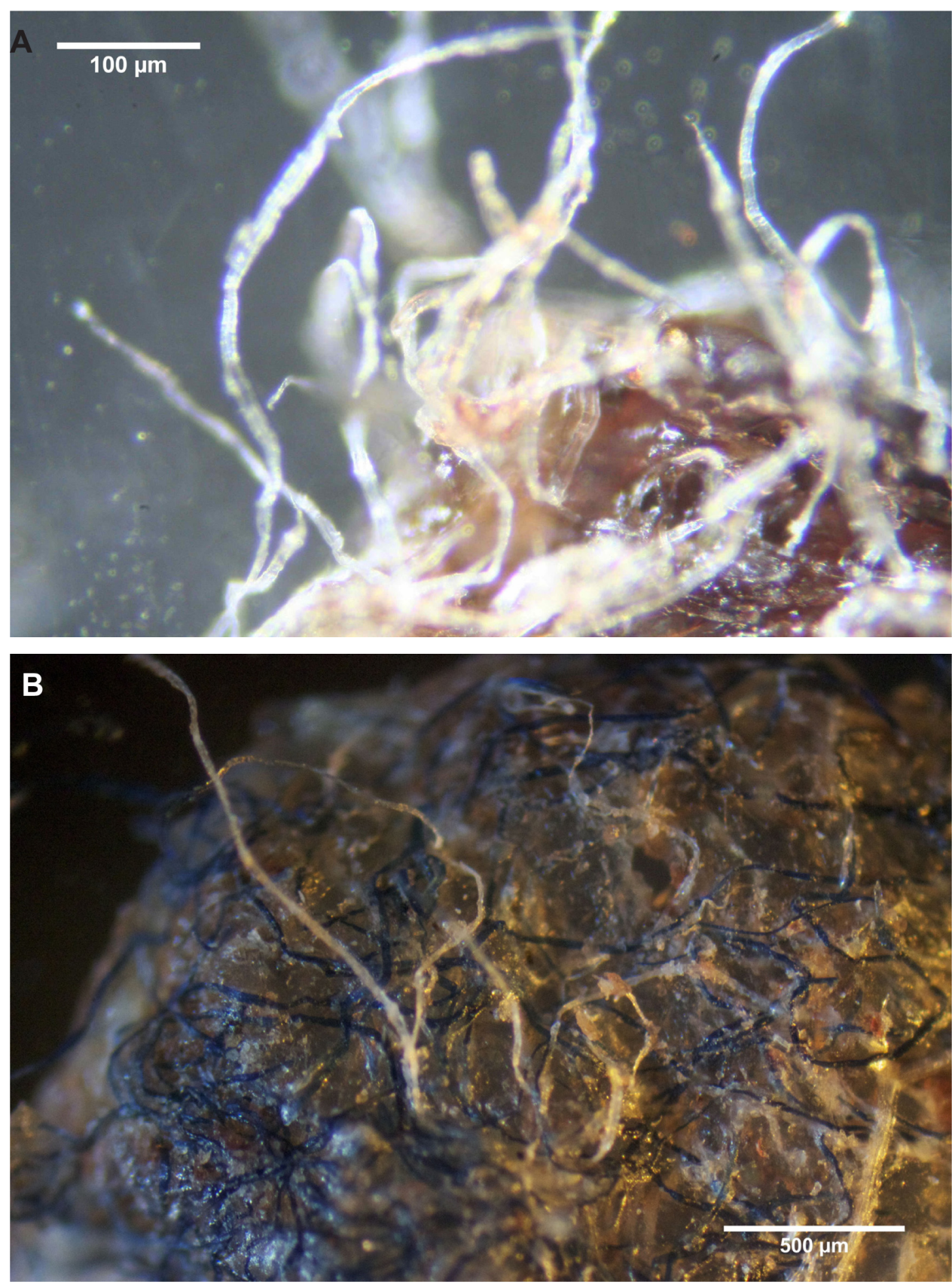

Figure I (Continued) 

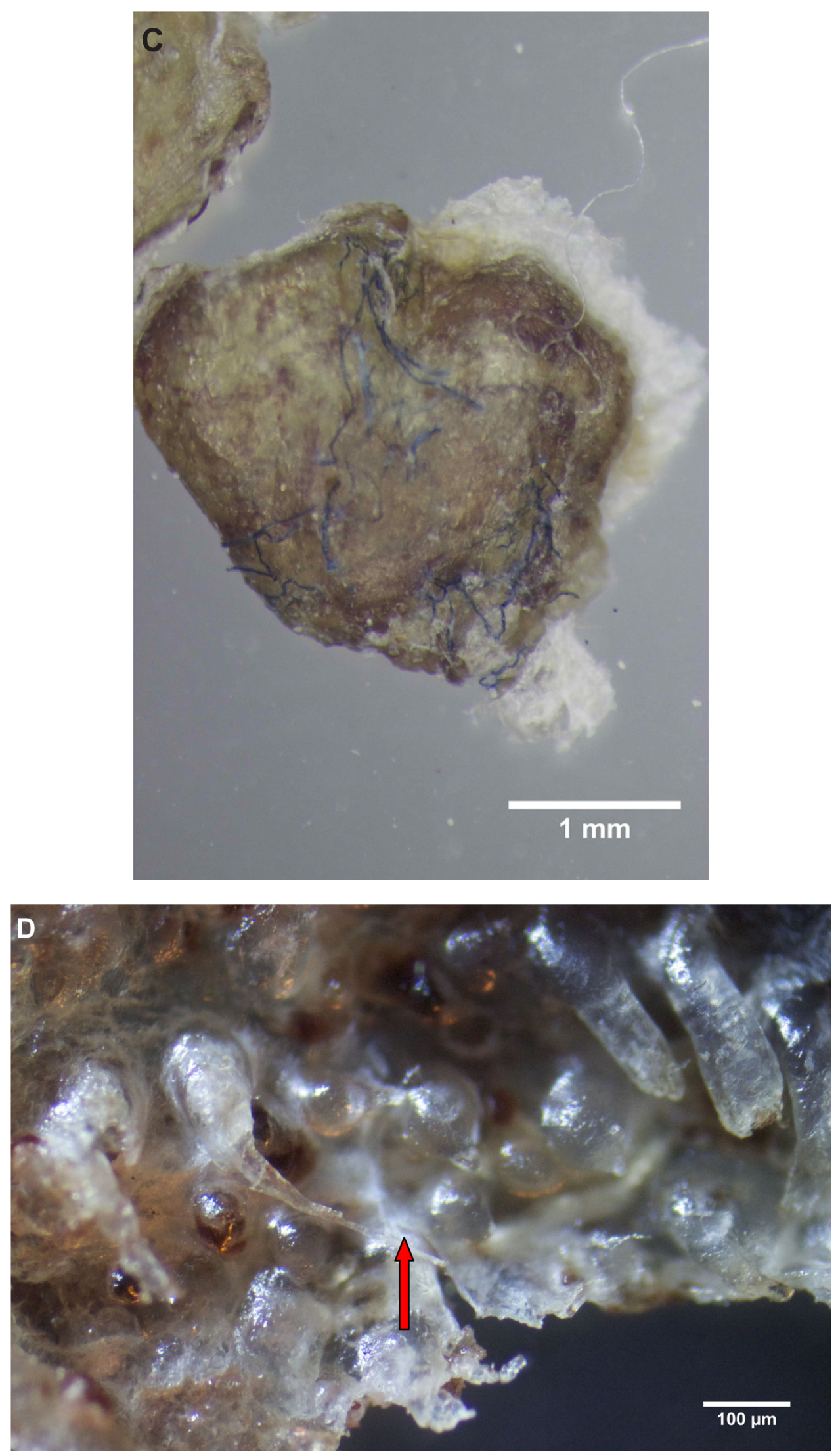

Figure I (A) Callus collected from patient I. Note hyaline filaments projecting from callus. 100x magnification. (B) Callus removed from patient 2. Note blue and hyaline filaments projecting from callus surface. 40× magnification. (C) Gross microscopic view of callus removed from patient 3 . Note blue filaments embedded in callus external surface. $8 \times$ magnification. (D) Callus from patient 2 demonstrating protruding keratin projections on internal surface. Note clear ingrown hair (arrow). 100× magnification. 

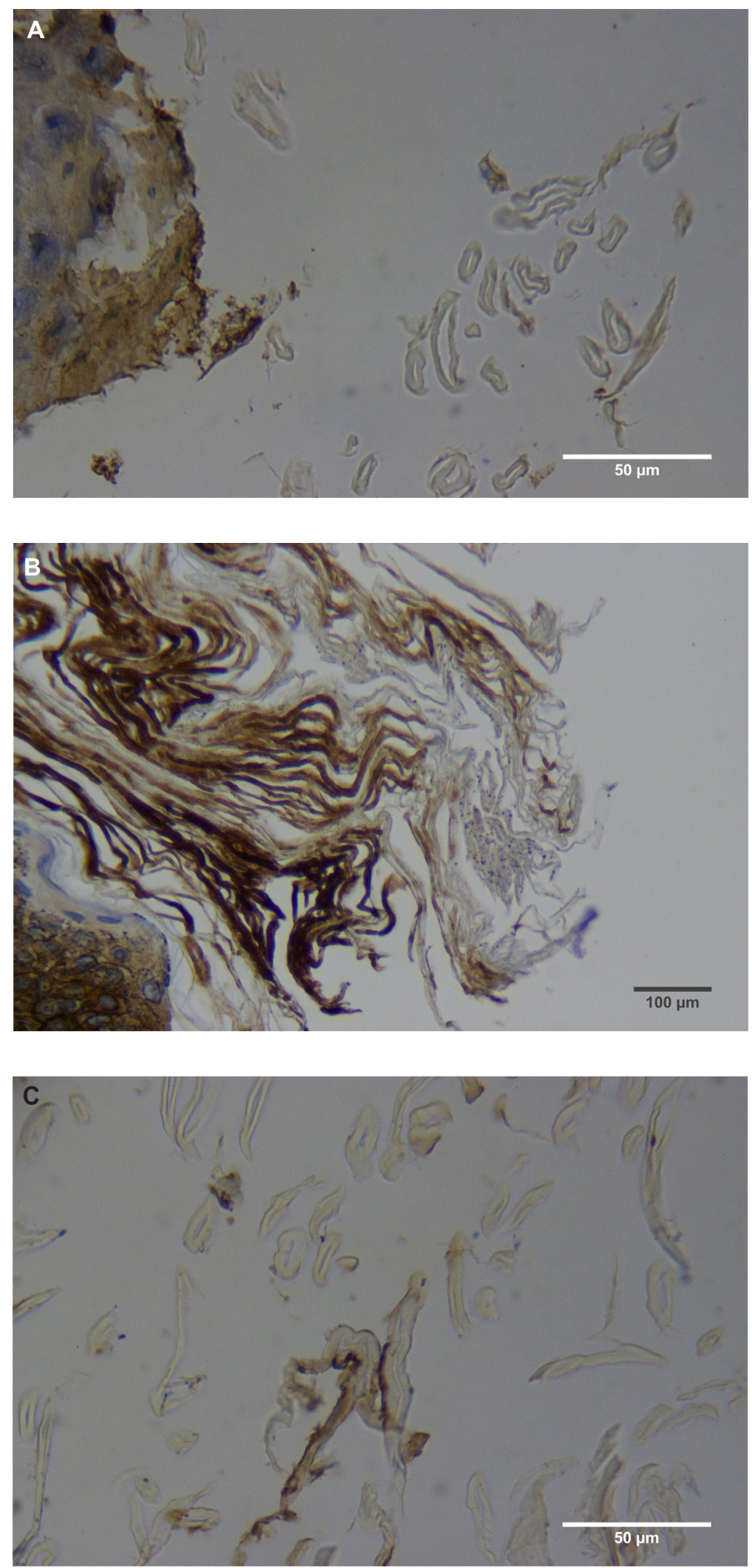

Figure 2 (Continued) 

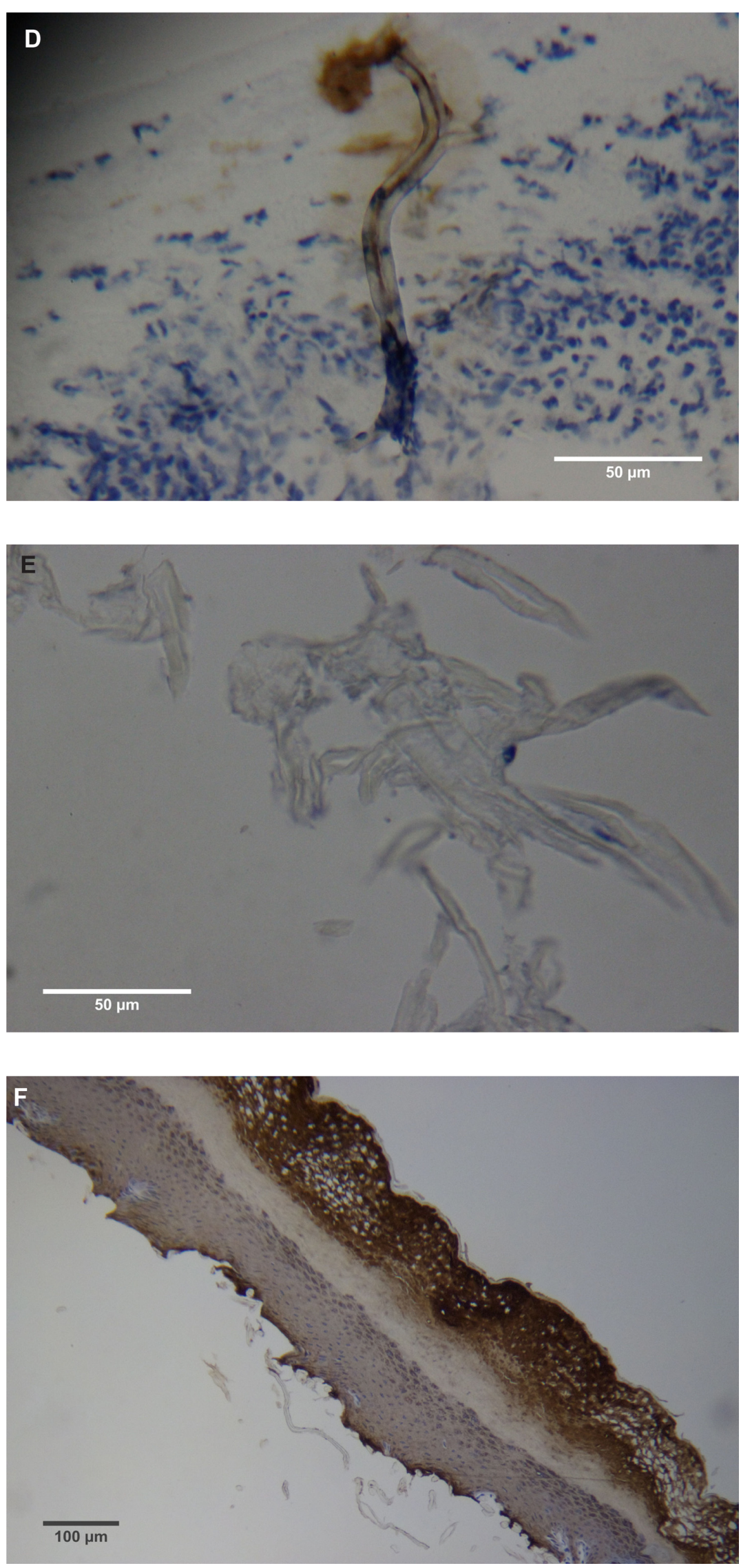

Figure 2 (Continued) 

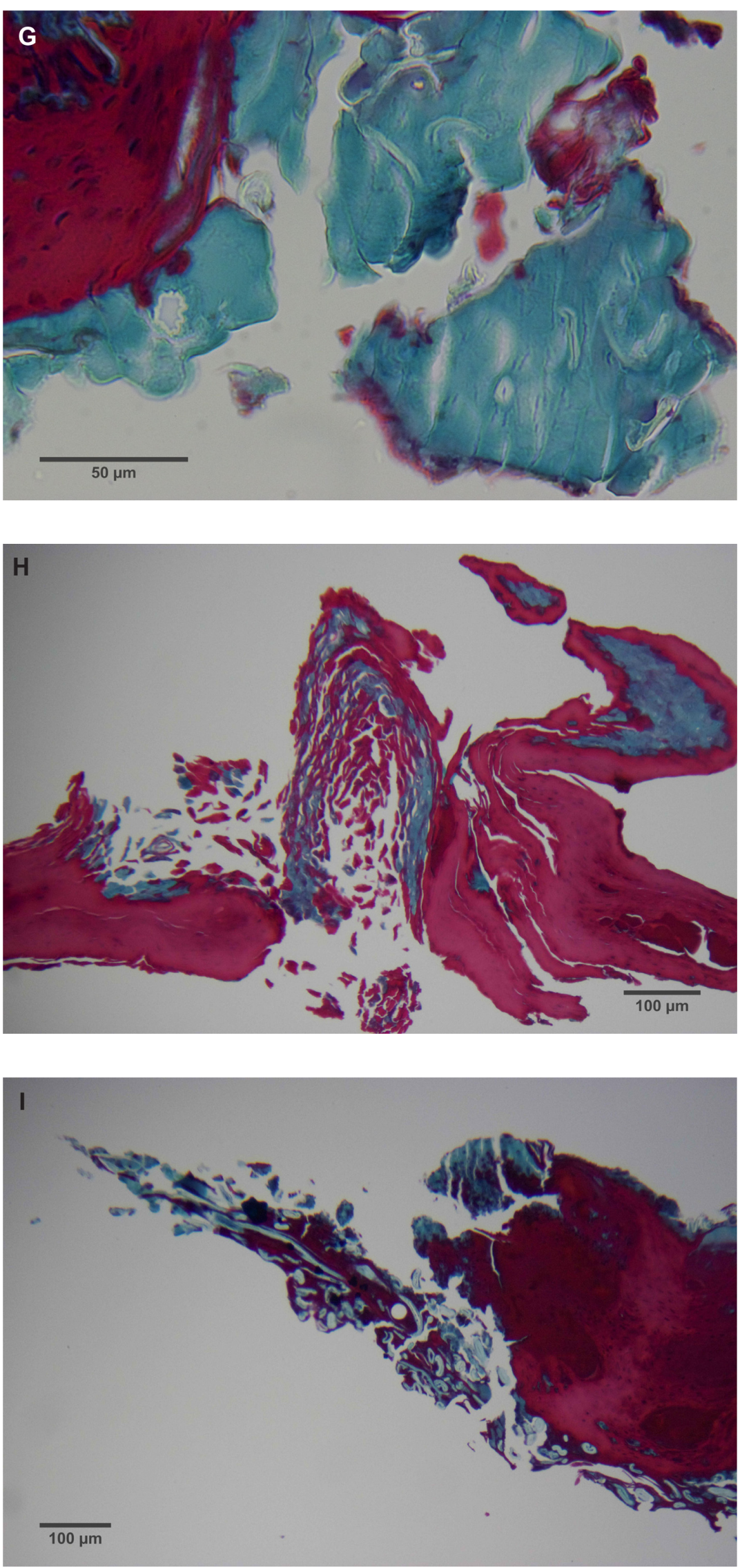

Figure 2 (Continued) 

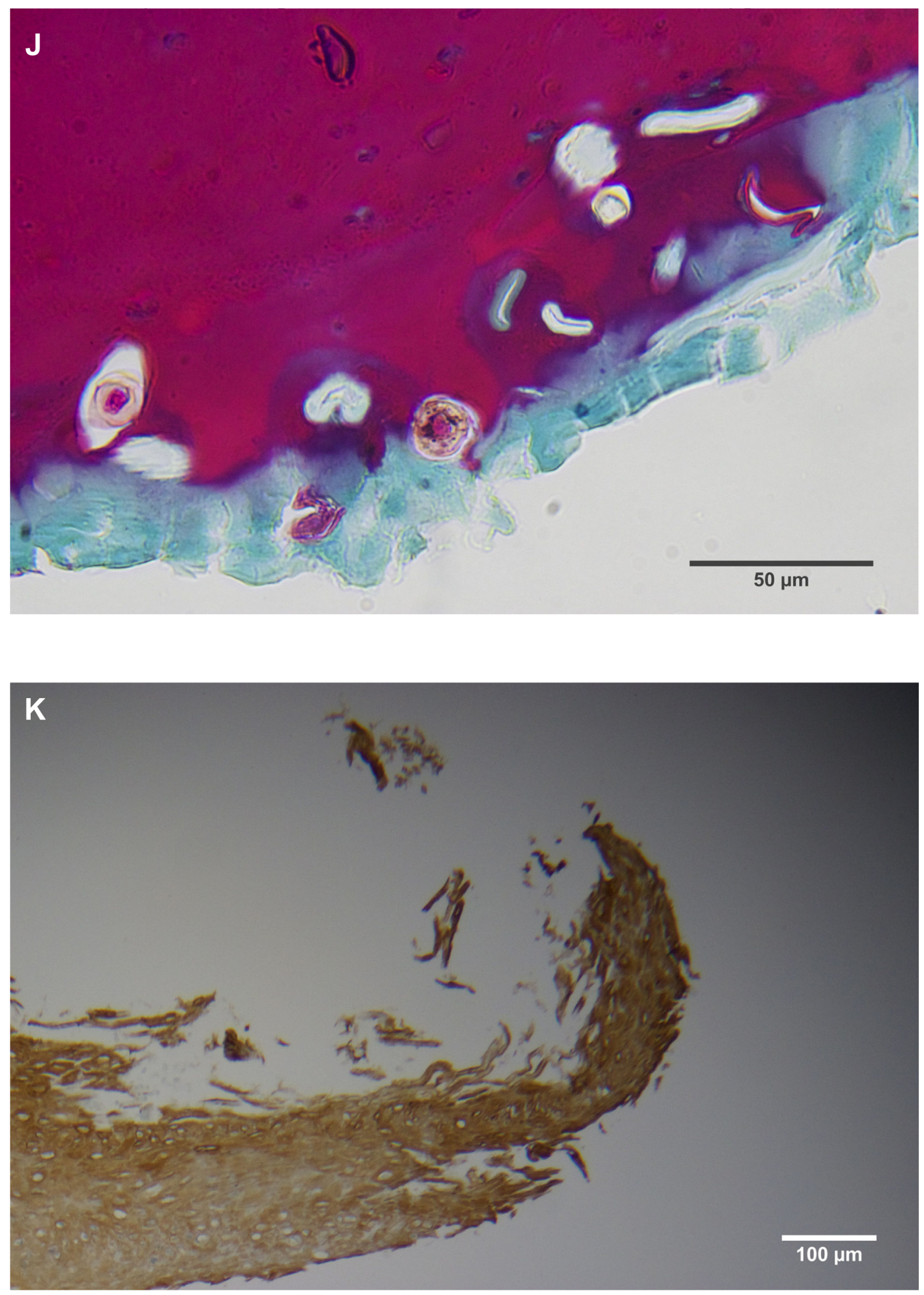

Figure 2 (Continued) 


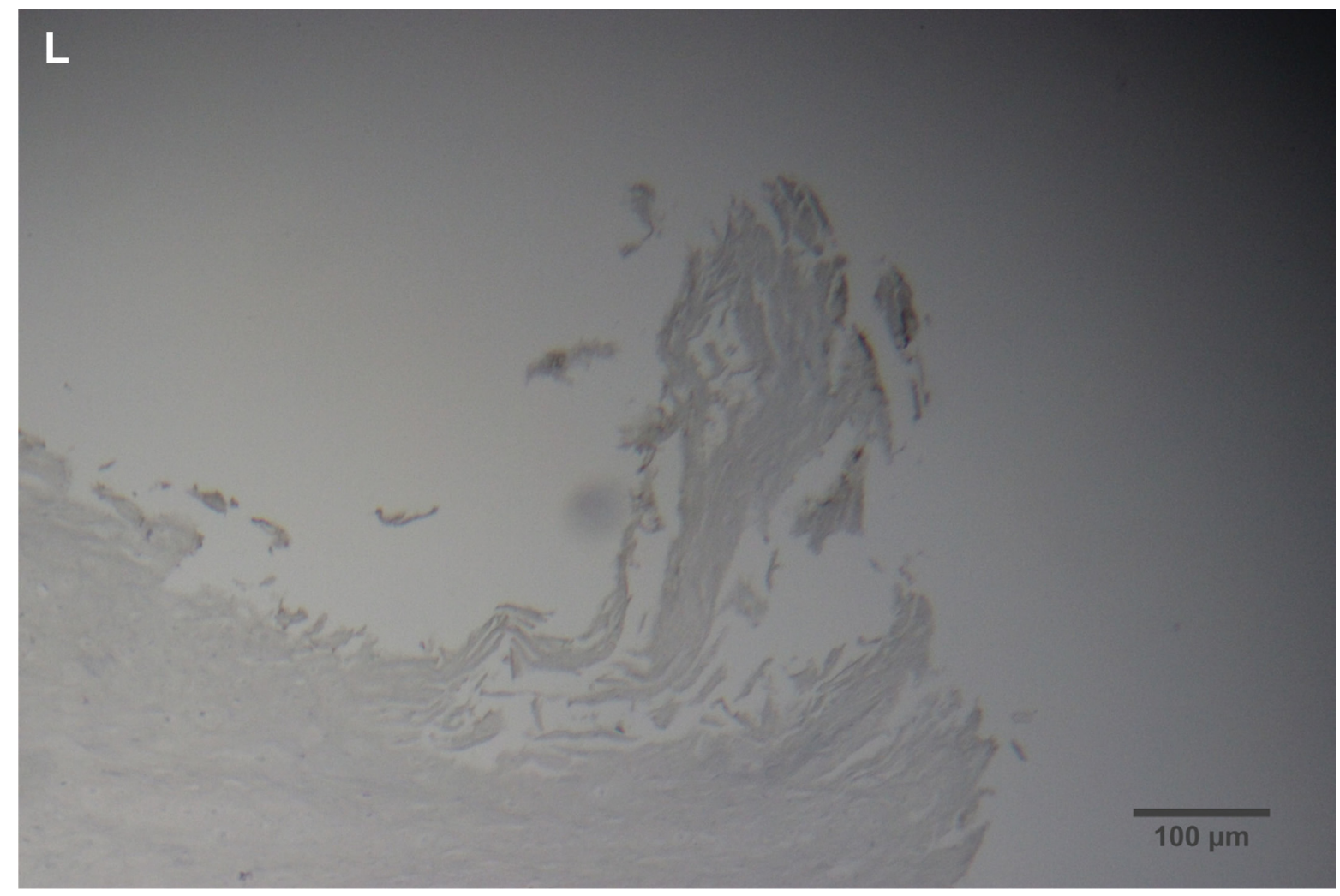

Figure 2 (A) Filament cross, oblique, and lateral sections, from a specimen collected from patient I showing round, elliptical/elongated, bean-like, or curved morphology. Note the hollow medulla and surrounding cortex. Cytokeratin (CK) AEI/AE3 staining. 100× magnification. (B) Specimen from patient I, demonstrating irregular, patchy keratin staining with CK AEI/AE3, longitudinal section. Note most filaments staining positive for keratin (dark brown). I00× magnification. (C) Specimen from patient I, demonstrating irregular, patchy keratin staining with CK AEI/AE3. Cross, oblique, and longitudinal sections. Note most filaments staining negatively for keratin. 400X magnification. (D) Longitudinal section of filament from patient I. Note retained nuclei, central medulla, and patchy keratin staining with CK AEI/AE3. 400 $\times$ magnification. (E) Sectioned filament from a specimen from patient I, which upon gross microscopic examination demonstrated floral-like or stellate formations. Note retained nuclei within tentacle-like filaments with tapered ends. CK AEI/AE3 staining. 400× magnification. (F) Sectioned callus from patient I, showing filaments stemming from the stratum basale with evolution inwards towards the dermis. Note filament sections with hollow medulla alongside the stratum basale. CK AEI/AE3 staining. I00X magnification. (G) Gömöri trichrome collagen-positive section of activated fibroblasts with filamentous inclusions from patient 2. Collagen stains green, keratin stains red. Growth of filaments was upwards toward the external surface. $400 \times$ magnification. $(\mathbf{H})$ Ruptured keratin projection from both external and internal surfaces from a specimen collected from patient 2, with areas of fibroblast proliferation stained green. Gömöri trichrome stain. 100× magnification. (I) Section from patient 2 stained with Gömöri trichrome, demonstrating both keratin (red) and collagen (green) filament cross-sections, with most filaments associated with the external callus surface. I00× magnification. (J) Gömöri trichrome stain of specimen from patient 2, demonstrating filaments in longitudinal, oblique, and cross-sections close to and within collagen-positive fibroblast collections (green). Note presence of filament cross-sections staining positively for keratin (red). 400× magnification. (K) Bovine digital dermatitis (BDD) filament, longitudinal section, showing positive CK AEI/ AE3 staining. 100× magnification. (L) BDD filament, longitudinal section, showing negative CK AE5/AE6 staining. 100× magnification.

$30 \mu \mathrm{m}$ long. They were present only in the interior areas of the sections and not in the stratum corneum. Numerous cocci were observed in the outermost layers of the stratum corneum of patient 2. Dieterle silver nitrate-based staining revealed bacteria consistent in size and shape with spirochetes in interior areas of histological sections in patients 1-4 (Figure 4D and E).

\section{BDD samples}

For comparison with MD samples, Warthin-Faulkner silver nitrate-based staining of proliferative-stage BDD samples was performed. Numerous spirochetes consistent in size with treponemes were detected among enlarged and ballooning keratinocytes (Figure 4F).

\section{Scanning electron microscopy MD filaments}

Under SEM, MD filaments from patients 1-4 varied from round and relatively smooth to tape-like, with an irregular, wrinkled, and frayed surface. Flatter specimens tended to be more wrinkled and frayed in appearance than rounder filaments. Some round filaments were slightly dimpled on the surface (Figure 5A). No hair-like scaling of the cortex was observed. Filaments from patient 1 were attached to an epithelial matrix and covering it in a carpet-like manner. Some filaments were tapered at the distal ends. Samples from patient 1 demonstrated a small amount of contamination with plant material that likely adhered to the specimen accidentally. Specimens collected from patients $2-4$ revealed 


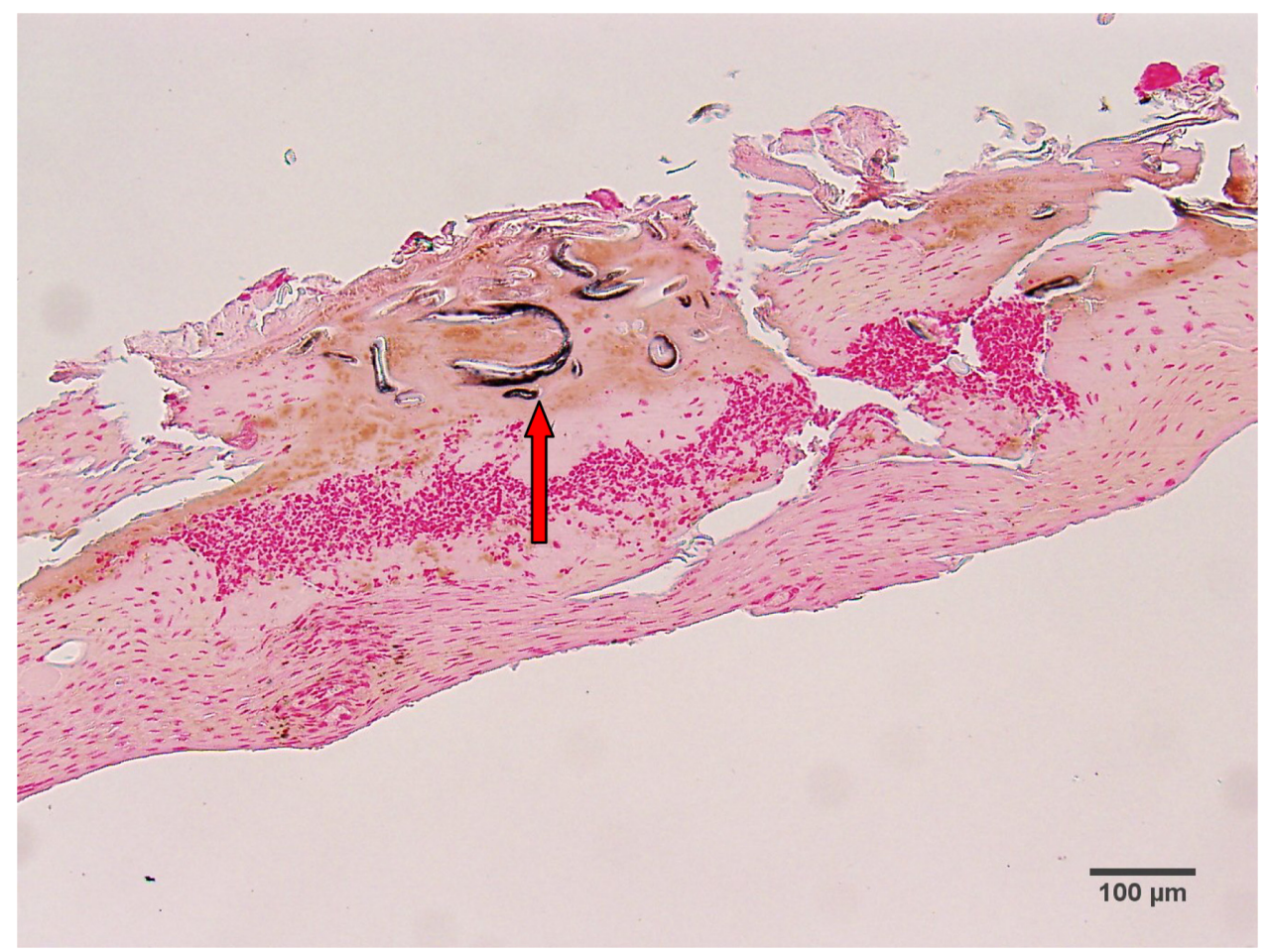

Figure 3 Fontana-Masson-stained section from patient 3, demonstrating positive melanin staining of blue filament sections (arrow). I00 $\times$ magnification.

filaments that were round to flattened, some of which lay underneath dermatological tissue and others protruding out of dermatological tissue.

\section{MD epithelial tissue}

A sample from patient 2 provided an image of a spirochete lying under epithelial tissue (Figure 5B). Other morphological forms of Borrelia spp could be seen with SEM (Figure 5C).

\section{BDD filaments}

Images of BDD samples revealed the keratin projections to be composed of long, stringy bundles of filamentous material or scaling, flaking material (Figure 5D).

\section{Transmission electron microscopy MD epithelial tissue}

Images of sections from patient 1 revealed numerous cocci, some of which displayed different stages of binary fission. Samples from patients 1 and 2 provided images consistent with different morphological forms of Borrelia in longitudinal and cross-section (data not shown).

\section{MD filaments}

Images of sections from patients 1, 2, and 4 revealed longitudinal and cross-sections of filaments (Figure 6A).
Cross-sections showed a cortex surrounding a hollow medulla that branched throughout the cortex. In longitudinal section, filaments were comprised of long filament bundles around a central medulla.

\section{BDD filaments}

Images of BDD filaments revealed spirochetes in longitudinal and cross-section (Figure 6B).

\section{Discussion}

We undertook this study to further characterize MD filaments and associated pathology. Initial histological studies done on calluses from MD patients revealed striking blue and red coloration in filaments projecting from these calluses (Figure 1A-D). These results are similar to the findings previously described in MD patients and demonstrate the consistency in MD pathology. ${ }^{4,5}$

Histological sectioning and electron microscopy revealed that MD filaments have a hollow medulla surrounded by a cortex, somewhat like a hair (Figures $2 \mathrm{~A}-\mathrm{E}$ and $6 \mathrm{~A}$ ). Association with hair follicles, the presence of follicularlike bulbs, and morphology such as scaling consistent with hairs has been reported in previous studies. ${ }^{4,5}$ Furthermore, preliminary SEM studies demonstrated that some colored filaments were small hairs. ${ }^{5}$ Hair-like morphology and keratin 

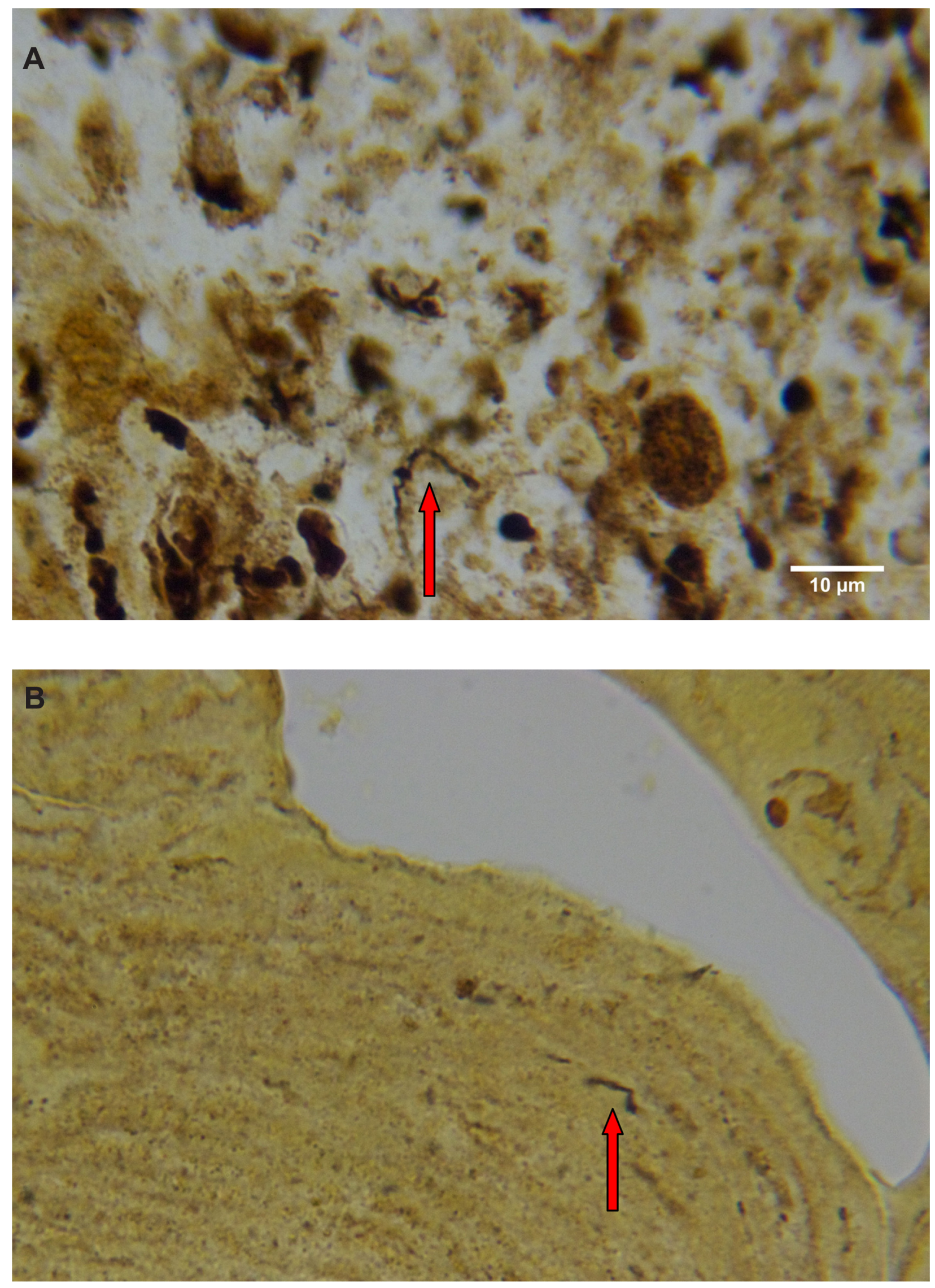

Figure 4 (Continued) 

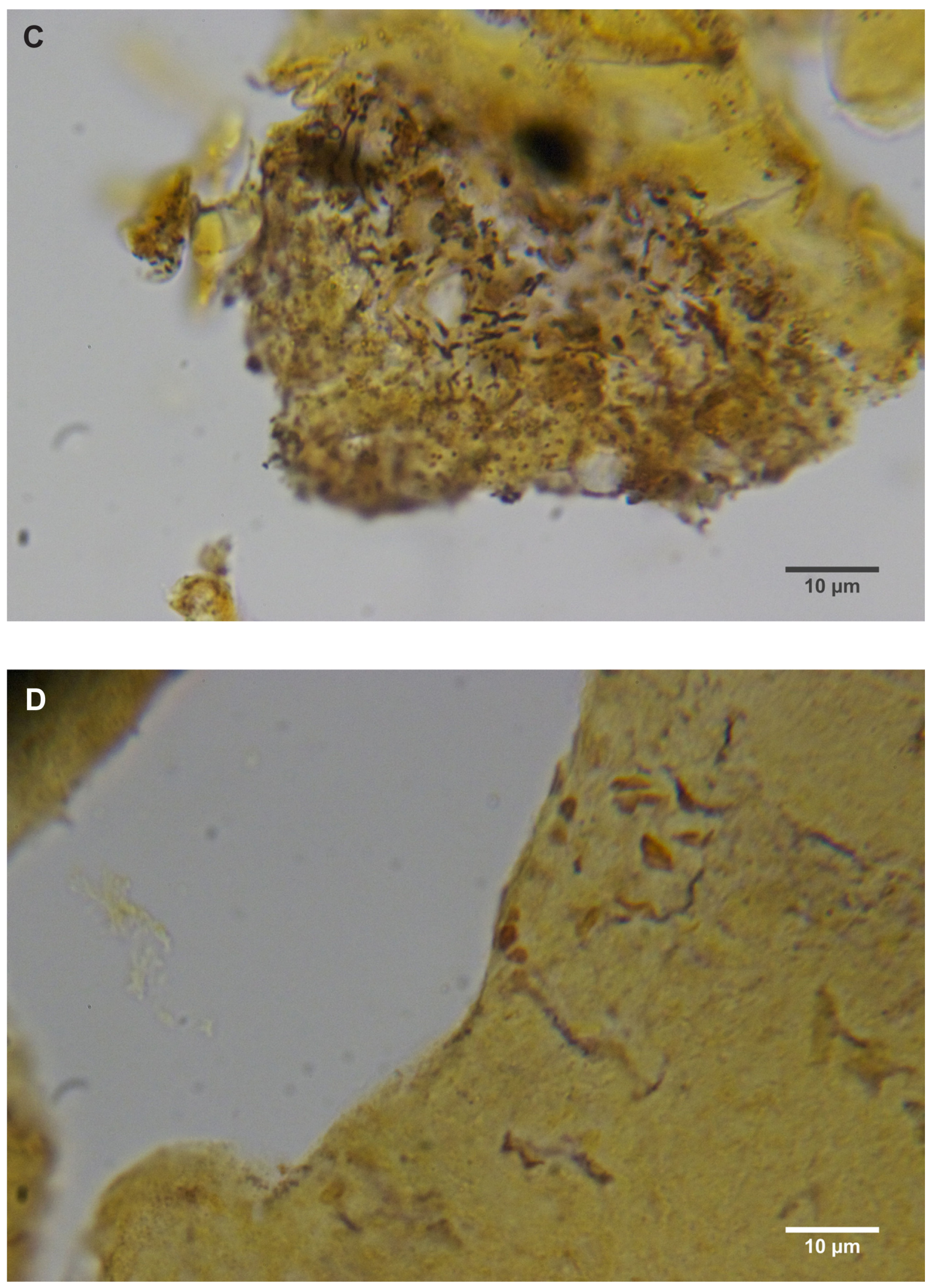

Figure 4 (Continued) 

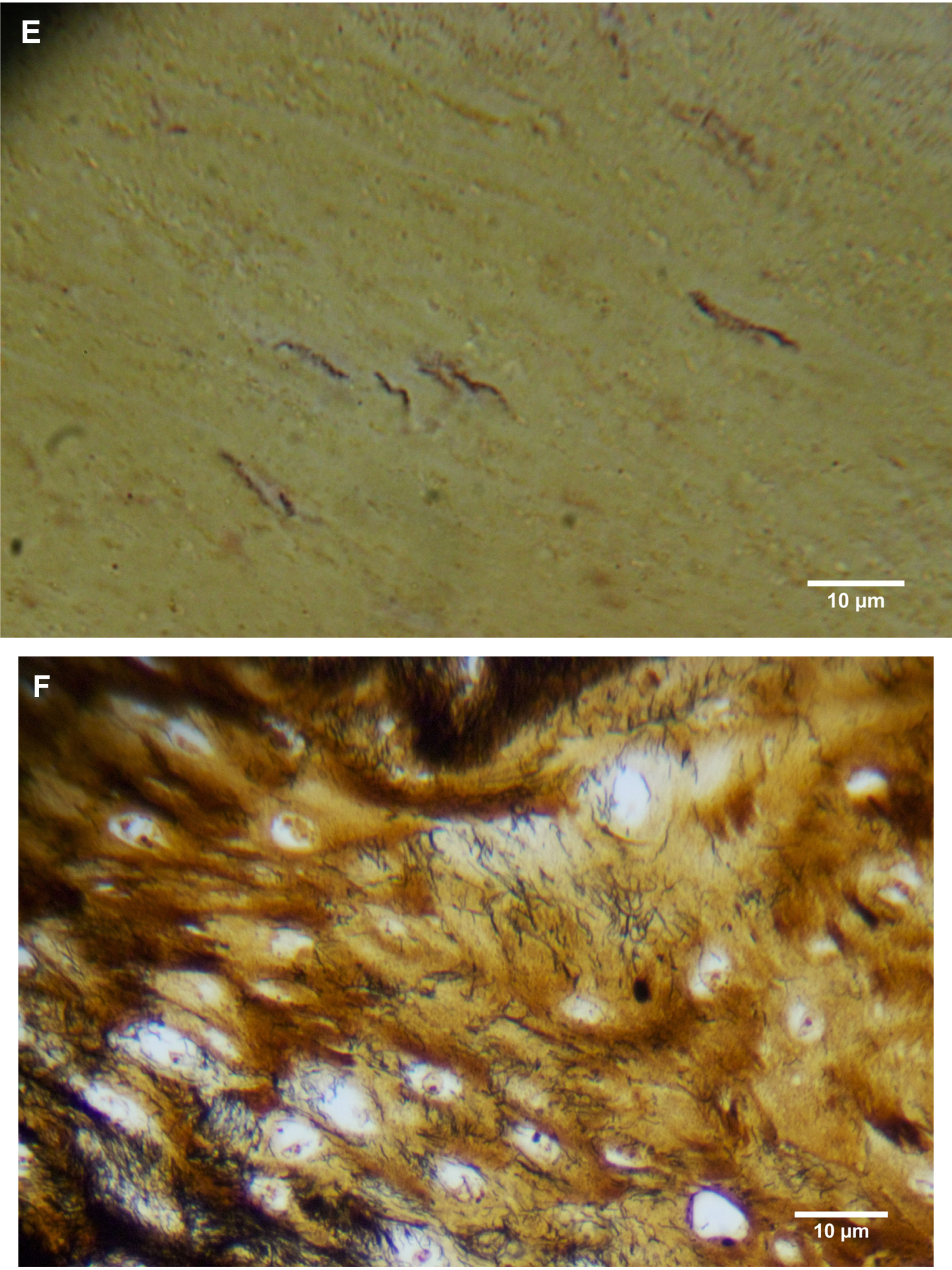

Figure 4 (A) Warthin-Starry-stained section of a Morgellons disease callus, patient 2, demonstrating a spirochete (arrow). I000× magnification. (B) Dieterle stain, spirochete from patient I (arrow). I000× magnification. (C) Dieterle stain, spirochetes from patient 2. 1000× magnification. (D) Dieterle stain, spirochetes from patient 3. 1000× magnification. (E) Dieterle stain, spirochetes from patient 4. 1000× magnification. (F) Warthin-Faulkner-stained bovine digital dermatitis section, showing numerous spirochetes among keratinocytes. $1000 \times$ magnification. 

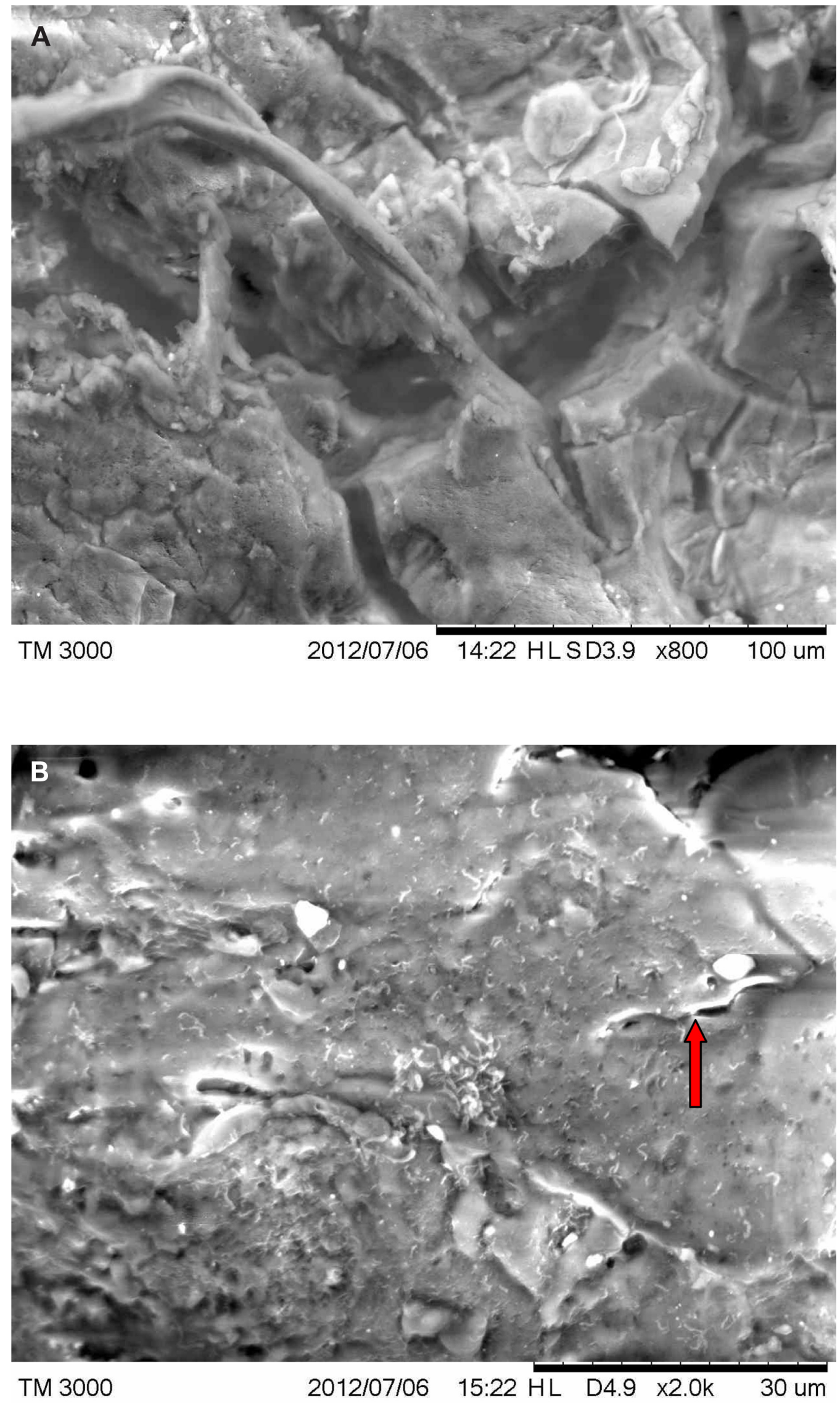

Figure 5 (Continued) 

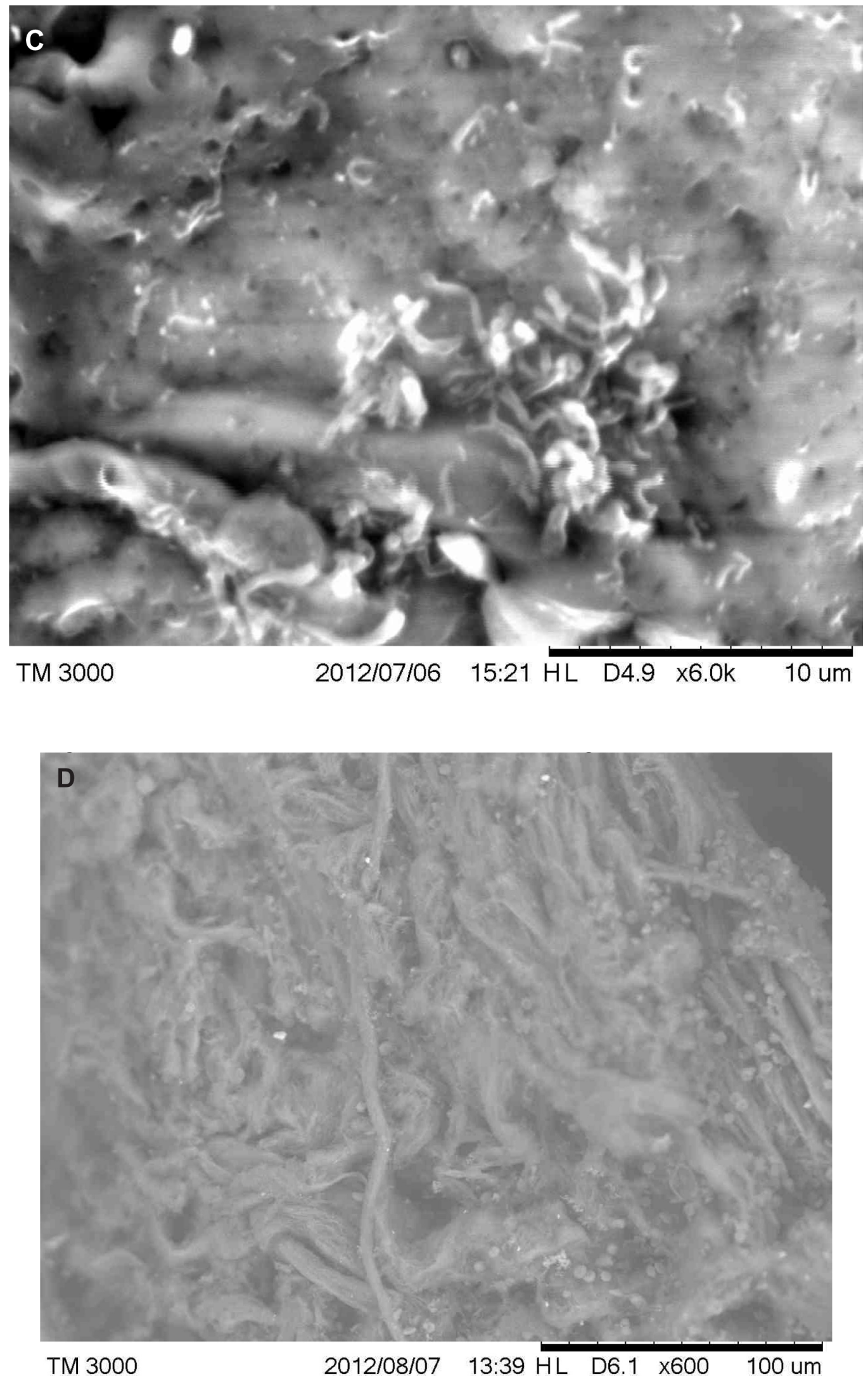

Figure 5 (A) Scanning electron microscopy (SEM), demonstrating typical appearance of filament projecting from skin surface, patient 2. Scale bar is shown at bottom of SEM. (B) SEM demonstrating the typical helical-shape outline of a spirochete under the skin surface, one-third down from top of right side (arrow). Scale bar is shown at bottom of SEM. (C) SEM of same specimen also demonstrating bacterial forms consistent with morphological variations of Borrelia spp. Scale bar is shown at bottom of SEM. (D) SEM of bovine digital dermatitis filaments, demonstrating filamentous and scaling surfaces. Scale bar is shown at bottom of SEM. 
composition of some filaments is supported by the fact that a minor portion of filaments in this study were composed exclusively of keratin. The possibility that some filaments of keratin composition are hairs is supported by our observation of normal-sized blue hairs with intact follicular bulbs in some MD specimens. ${ }^{4,5}$

Previous studies confirmed the presence of keratin in MD filamentous tissue. ${ }^{5}$ However, CK AE1/AE3 keratin staining of filaments associated with MD in this study was most often inconsistent and patchy, and many filaments did not demonstrate hair-like scaling, even under SEM (Figure 2A-F and 5D). Filaments ranged from round and relatively smooth to flattened with wrinkled, fraying, or eroded surfaces, and this morphology is more consistent with collagen composition than with keratin composition.
This finding led us to investigate the possibility of collagen composition of filaments by histochemistry and electron microscopy. Gömöri trichrome staining revealed that the MD filaments observed in this study have a collagen component (Figure 2G-J). Filaments in cross-section and longitudinal section were also found adjacent to collagenpositive staining areas of epithelial tissue. Ingrown keratin projections often contained collections of proliferating fibroblasts that were associated with filament formation (Figure 2G-J). Retention of nuclei within filaments was frequently observed in filaments of both keratin and collagen composition. These findings provide evidence that filaments are cellular in origin, resulting from activation, proliferation, and elongation of both keratinocytes and fibroblasts.

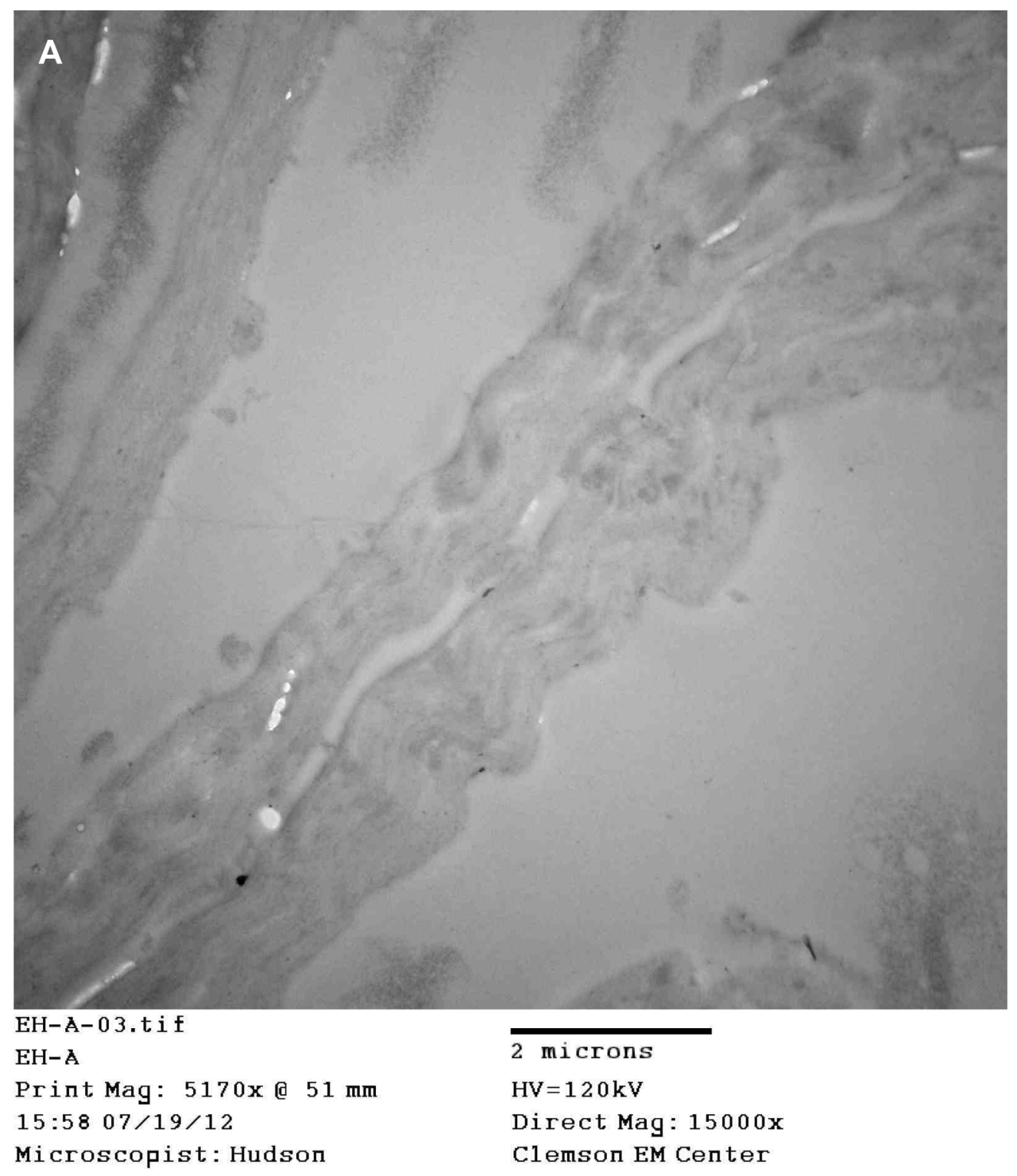

Figure 6 (Continued) 


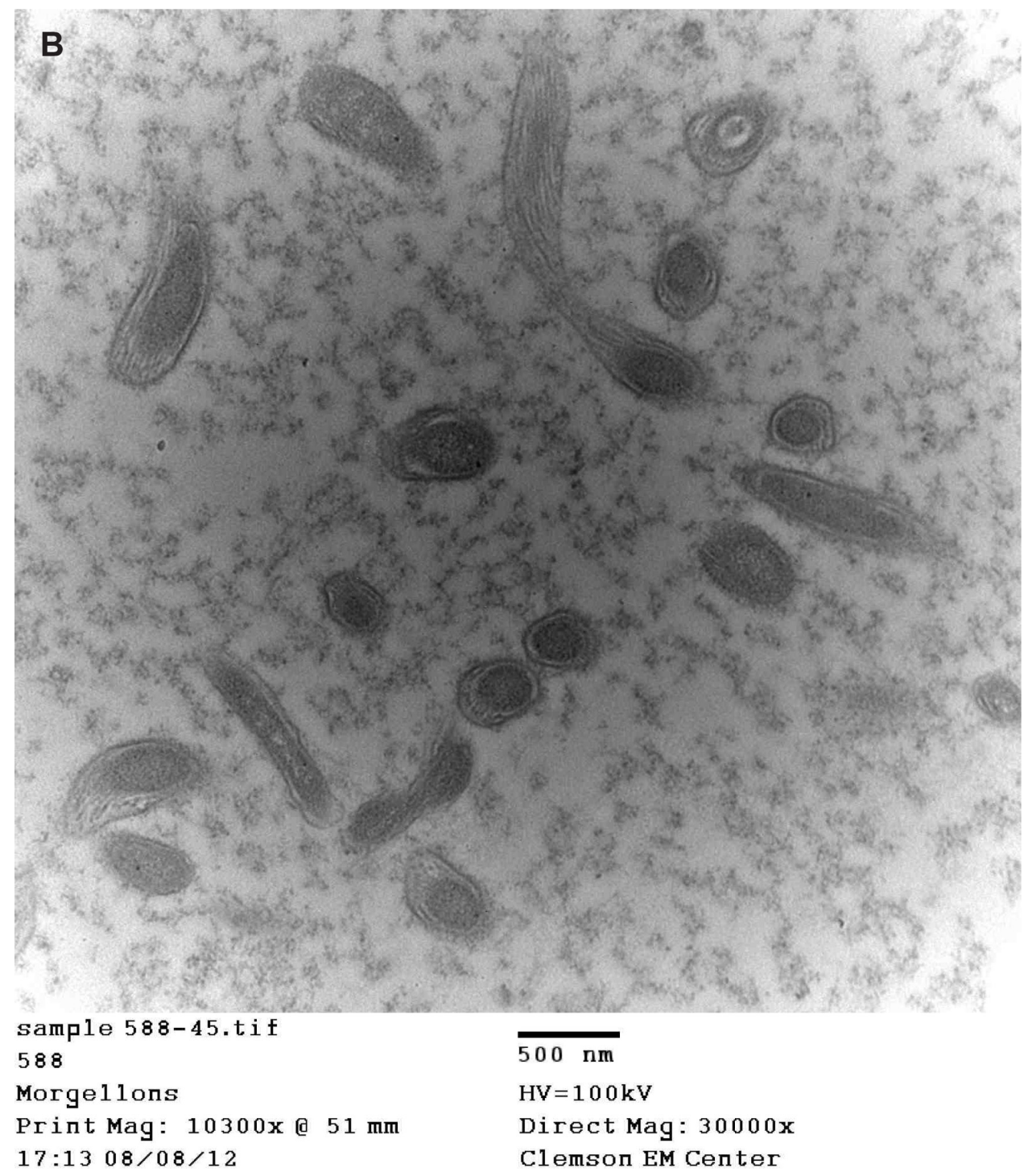

Figure 6 (A) Transmission electron microscopy (TEM), demonstrating longitudinal section of filament from patient 4 . Note hollow medulla surrounded by a cortex. Scale bar is shown at bottom of TEM. (B) TEM of bovine digital dermatitis (BDD) section showing both longitudinal and cross sections of spirochetes. Scale bar is shown at bottom of TEM.

Prior to this study, there was speculation that MD filament coloration could be structural in nature rather than produced by pigmentation. This initial hypothesis was based on the assumption that the filaments were keratin in composition. However, because bluish coloration of skin has been described in patients with pinta due to T. carateum infection, ${ }^{17}$ we speculated that altered melanin expression could be responsible for the blue and red coloration. Fontana-Masson histological staining confirmed that the blue color seen in filaments was caused, at least in part, by melanin pigmentation (Figure 3). Fontana-Masson melanin staining was negative in red filaments, and therefore the underlying cause of this coloration remains to be elucidated. It is possible that red coloring of filaments could involve pigmentation with a heme-containing compound or some other erythroid substance.
In our investigation, spirochetes were detected in MD dermatological specimens using Warthin-Starry and/or Dieterle silver nitrate-based histological staining (Figure 4A-E) and electron microscopic techniques (Figure 5B and C). A similar animal dermopathy, BDD, is also associated with unusual keratin filament production and the presence of spirochetes, as confirmed in our study (Figures 4F and 6B). In the case of BDD, detected spirochetes belong to various treponemal species..$^{7-11}$ The genetic identification of MD spirochetes remains to be determined, but the fact that patients with MD have a high rate of tickborne illness suggests that the detected spirochetes are from the genus Borrelia, ${ }^{3}$ and recent molecular studies provide evidence that MD cases are associated with B. burgdorferi sensu stricto. ${ }^{19}$ The current findings suggest that in MD, 
the presence of spirochetes not only stimulates and alters keratin expression by keratinocytes in the epidermis but also causes increased collagen expression by proliferative activated fibroblasts. Although our study was too small to draw definitive conclusions about a spirochetal etiology of $\mathrm{MD}$, the histopathological results are provocative, and further testing in larger numbers of MD patients is warranted.

Various pathogens are known to induce hyperproliferative dermopathy. Hyperplasia, hyperkeratosis and keratin filament production is characteristic of hairy leukoplakia associated with Epstein-Barr virus infection, ${ }^{20}$ while overexpression of collagen (amianthroid) fibers in inflammatory myofibroblastic tumors has been associated with EpsteinBarr virus and Kaposi sarcoma-associated herpes virus infection. ${ }^{21}$ MD lesions are histopathologically distinct, however, from both hairy leukoplakia and myofibroblastic tumors. Increased keratin expression caused by upregulation of keratinocyte growth factor/fibroblast growth factor-7 has been reported in cholesteatoma, a hyperproliferative epithelial disease. ${ }^{22}$ As a consequence of $B$. burgdorferi infection and an inflammatory environment, dermal fibroblasts may become activated, modulating epithelial growth and differentiation through increased keratinocyte growth factor/fibroblast growth factor-7 expression. ${ }^{23}$ More importantly, B. burgdorferi has been shown to attach to and invade human fibroblasts, and viable B. burgdorferi spirochetes have been isolated from lysates of fibroblast monolayers after antibiotic therapy. ${ }^{24}$ This suggests that the spirochetes associated with MD may act similarly, causing unusual collagen and keratin filament production. It also suggests that persistent infection despite antibiotic treatment could be problematic in this subgroup of patients with Lyme-like illness.

A recent study from the Centers for Disease Control and Prevention (CDC) concluded that most of the filaments collected from MD dermatological lesions were cellulose, likely of cotton origin. ${ }^{12}$ In contradiction to their own conclusion, however, the authors of the CDC study reported that material collected from MD patients was over $83 \%$ protein in composition and subsequently that collected material was "likely superficial skin or cellulose consistent with cotton fibers." 12 This sample composition is consistent with keratin or other proteins rather than cellulose. The conclusions of the CDC study are contradictory, because cellulose is a carbohydrate rather than a protein and if $83 \%$ of samples were composed of protein, then only a minor percentage of samples (17\% at most) could exclusively be composed of cellulose. The protein findings can be better explained by the possibility that the major proportion of patient samples included keratin and collagen fibers, while a minor portion of the samples included nonprotein debris that accidentally adhered to open wounds.

In summary, histological observations and electron microscopic imaging from representative MD samples suggest that dermal filaments are composed of keratin and collagen and result from proliferation and activation of keratinocytes and fibroblasts in the epidermis. We have also confirmed the presence of spirochetes in these samples. Although the number of patients in our detailed histopathological study is too small to draw definitive conclusions about the etiology of MD in general, the interplay of MD filaments and spirochetal infection merits further study.

\section{Acknowledgments}

The authors thank Drs Stewart Adams, Gordon Atkins, Robert Bransfield, Douglas Demetrick, Dorte Dopfer, JoAnn Hudson, Alan MacDonald, Elizabeth Rasmussen, Eva Sapi, Virginia Savely, Matthew Shawkey, Janet Sperling, and Michael Sweeney for helpful discussion. We thank Dr Robert B Allan for technical support and Lorraine Johnson for manuscript review, and we are grateful to Harriet Bishop and Cindy Casey for providing firsthand information about Morgellons disease. Partial research funding was provided by the Charles E Holman Foundation, Austin, TX, USA.

\section{Disclosure}

RBS serves without compensation on the medical advisory panel for QMedRx Inc. He has no financial ties to the company. MJM, PJM, and RBS serve without compensation on the scientific advisory panel of the Charles E Holman Foundation. DGK has no conflicts to declare.

\section{References}

1. Savely VR, Leitao MM, Stricker RB. The mystery of Morgellons disease: infection or delusion? Am J Clin Dermatol. 2006;7:1-5.

2. Savely VR, Leitao MM. Skin lesions and crawling sensations: disease or delusion? Adv Nurse Pract. 2005;13:16-17.

3. Savely VR, Stricker RB. Morgellons disease: analysis of a population with clinically confirmed microscopic subcutaneous fibers of unknown etiology. Clin Cosmet Investig Dermatol. 2010;3:67-78.

4. Middelveen MJ, Stricker RB. Filament formation associated with spirochetal infection: a comparative approach to Morgellons disease. Clin Cosmet Investig Dermatol. 2011;4:167-177.

5. Middelveen MJ, Rasmussen EH, Kahn DG, Stricker RB. Morgellons disease: a chemical and light microscopic study. J Clin Exp Dermatol Res. 2012;3:140.

6. Cheli R, Mortellaro CM. Digital dermatitis in cattle. In: World Association for Buiatrics. Proceedings of the 8th International Meeting on Diseases of Cattle; September 9-13, 1974; Milan, Italy. Piacenza, Italy: Piacentina Gallarati; 1974:208-213. 
7. Borgmann JE, Bailey J, Clark EG. Spirochete-associated bovine digital dermatitis. Can Vet J. 1996;37:35-37.

8. Walker RL, Read DH, Loretz KJ, Hird DW, Berry SL. Humoral response of dairy cattle to spirochetes isolated from papillomatous digital dermatitis lesions. Am J Vet Res. 1997;58:744-748.

9. Read DH, Walker RL. Papillomatous digital dermatitis (footwarts) in California dairy cattle: clinical and gross pathologic findings. $J$ Vet Diagn Invest. 1998;10:67-76.

10. Evans NJ, Brown JM, Demirkan I, et al. Association of unique, isolated treponemes with bovine digital dermatitis lesions. J Clin Microbiol. 2009;47:689-696.

11. Döpfer D, Anklam K, Mikheil D, Ladell P. Growth curves and morphology of three Treponema subtypes isolated from digital dermatitis in cattle. Vet J. 2012;193:685-693.

12. Pearson ML, Selby JV, Katz KA, et al. Clinical, epidemiologic, histopathologic and molecular features of an unexplained dermopathy. PLoS One. 2012;7:e29908.

13. Harvey WT. Morgellons disease. J Am Acad Dermatol. 2007;56: 705-706.

14. Harvey WT, Bransfield RC, Mercer DE, Wright AJ, Ricchi RM, Leitao MM. Morgellons disease, illuminating an undefined illness: a case series. J Med Case Rep. 2009;3:8243.

15. Hylwa SA, Bury JE, Davis MD, Pittelkow M, Bostwick JM. Delusional infestation, including delusions of parasitosis: results of histologic examination of skin biopsy and patient-provided skin specimens. Arch Dermatol. 2011;147:1041-1045.

16. Stricker RB, Middelveen MJ. Morgellons disease: more questions than answers. Psychosomatics. 2012;53:504-505.
17. Farnsworth N, Rosen T. Endemic treponematosis: review and update Clin Dermatol. 2006;24:181-190.

18. D'Alba L, Kieffer L, Shawkey MD. Relative contributions of pigments and biophotonic nanostructures to natural color production: a case study in budgerigar (Melopsittacus undulatus) feathers. $J$ Exp Biol. 2012;215(Pt 8):1272-1277.

19. Middelveen MJ, Poruri A, Mayne PJ, Sapi E, Kahn DG, Stricker RB Association of Borrelia burgdorferi infection with Morgellons disease. J Invest Med. 2013;61:225.

20. DeSousa YG, Freese UK, Greenspan D, Greenspan JS. Diagnosis of Epstein-Barr virus infection in hairy leukoplakia by using nucleic acid hybridization and noninvasive techniques. J Clin Microbiol. 1990; 28: 2775-2778.

21. Kandemir NO, Barut F, Ekinci T, Karagülle C, Ozdamar SO. Intranodal palisaded myofibroblastoma (intranodal hemorrhagic spindle cell tumor with amianthoid fibers): a case report and literature review. Diagn Pathol. 2010;5:12.

22. Raynov AM, Choung YH, Park HY, Choi SJ, Park K. Establishment and characterization of an in vitro model for cholesteatoma. Clin Exp Otorhinolaryngol. 2008;1:86-91.

23. Raffa S, Leone L, Scrofani C, Torrisi MR, Barbara M. Cholesteatomaassociated fibroblasts modulate growth and differentiation through KFG/FGF7 secretion. Histochem Cell Biol. 2012;138:251-269.

24. Klempner MS, Rogers RA, Noring R. Invasion of fibroblasts by the Lyme spirochete Borrelia burgdorferi. J Infect Dis. 1993;167:1074-1081.
Clinical, Cosmetic and Investigational Dermatology

\section{Publish your work in this journal}

Clinical, Cosmetic and Investigational Dermatology is an international, peer-reviewed, open access, online journal that focuses on the latest clinical and experimental research in all aspects of skin disease and cosmetic interventions. All areas of dermatology will be covered; contributions will be welcomed from all clinicians an

\section{Dovepress}

basic science researchers globally. This journal is indexed on CAS The manuscript management system is completely online and includes a very quick and fair peer-review system, which is all easy to use. Visit http://www.dovepress.com/testimonials.php to read real quotes from published authors. 